\title{
Morphological Alterations in Megakaryocytes in Bone Marrow Aspirate of Thrombocytopenia Cases
}

Sharma R, Kiran $\mathbf{C M}^{*}$ and Ramdas A

Department of Pathology, Pondicherry Institute of Medical Sciences, Pondicherry, India

\begin{abstract}
Background: Thrombocytopenia is seen in various haematological and non haematological conditions and has been defined as platelet count less than $1.5 \mathrm{lakh} / \mathrm{mm}^{3}$. Dysplastic changes are well known in Myelo-Dysplastic Syndrome (MDS) but these can be seen in other haematological conditions.
\end{abstract}

Methods: This was a retrospective study done on 100 bone marrow aspirations in a tertiary care center in South India from Jan 2015 to Dec 2016. All bone marrow aspirations in this study period was retrieved and the slides were reviewed by two pathologists.

Results: Total of 100 cases were included in the study. Out of 100 cases of bone marrow aspiration, 78 cases were of thrombocytopenia, 6 cases were of thrombocytosis, and 16 cases were of normal platelet count. The most common cause for which bone marrow aspiration was sought was pancytopenia (30\%), followed by ITP (15\%), PUO $(12 \%)$, Anaemia (10\%). Bicytopenia (8\%), Hepatosplenomegaly (3\%), Lympho-proliferative disorder (5\%), GMCSF treatment (1\%), MPN (3\%), T.B, MM (4\% each), MDS (2\%), AL (2\%), MGUS (1\%).

Conclusion: Most common cause of thrombocytopenia in this study was pancytopenia followed by ITP Dysplastic features were seen in MDS but also present in other conditions like megaloblastic anaemia and acute leukemia.

Keywords: Megakaryocytes; Morphology; Thrombocytopenia

Abbreviations: ITP: Immune Thrombocytopenic Purpura; PUO: Pyrexia of Unknown Origin; GMCSF: Granulocyte Monocyte Colony Stimulating Factor; MPN: Myelo-Proliferative Neoplasm; MM: Multiple Myeloma; MDS: Myelodysplastic Syndrome; AL: Acute Leukemia; MGUS: Monoclonal Gammopathy of Undetermined Significance

\section{Introduction}

Megakaryocytes are the myeloid cells that are present mainly in the bone marrow but can be present in lung and peripheral blood [1]. They arise from pleuripotent hematopoietic stem cells under the influence of thrombopoietin. Thrombocytopenia is seen in various haematological and non haematological conditions and has been defined as platelet count less than $1.5 \mathrm{lakh} / \mathrm{mm}^{3}$ [2]. Dysplastic changes are well known in Myelo-Dysplastic Syndrome (MDS) but these can be seen in other haematological conditions [2]. This study was undertaken for better understanding of dysplastic megakaryocytic alterations in MDS and non MDS cases and their contribution to thrombocytopenia in non MDS conditions so as to increase diagnostic accuracy.

\section{Materials and Methods}

This was a retrospective study done on 100 bone marrow aspirations in a tertiary care center in South India from Jan 2015 to Dec 2016. All bone marrow aspirations in this study period were retrieved and the slides were reviewed by two pathologists (Table 1). The clinical details, complete blood count and other relevant investigations were reviewed from the case files. The aspirate was studied to assess the number and morphology of megakaryocytes. The number of megakaryocytes were considered normal (1 megakaryocyte/1-3 Low Power Field), increased (greater than 2 megakaryocytes/low power field), decreased (1 megakaryocytes/5-10) [3]. The morphological changes i.e nuclear segmentation, presence of immature forms, dysplastic forms, micro-megakaryocytes, emperipolesis, platelet budding, cytoplasmic vacuolations, bare megakaryocytes and hyopo-granular forms were studied. The presence of abnormal megakaryocytes which included the micro-megakaryocytes, dysplastic forms, megakaryocytes with separated lobes and hypogranular forms were considered as dysmegakaryocytopoiesis (Figure 1). Normal megakaryocytes were considered to have four to sixteen nuclear lobes. Immature megakaryocytes were defined as young forms of megakaryocytes with

\begin{tabular}{|l|c|}
\hline Bone marrow aspiration & No. of cases \\
\hline Pancytopenia & 30 \\
\hline ITP & 15 \\
\hline PUO & 12 \\
\hline Anaemia & 10 \\
\hline Bicytopenia & 8 \\
\hline Hepatosplenomegaly & 3 \\
\hline Lymphoproliferative disorder & 5 \\
\hline GMCSF treatment & 1 \\
\hline MPN & 3 \\
\hline AL & 2 \\
\hline Multiple Myeloma & 4 \\
\hline Tuberculosis & 4 \\
\hline MGUS & 1 \\
\hline
\end{tabular}

Table 1: Showing causes of thrombocytopenia.

*Corresponding author: Kiran CM, Department of Pathology, Pondicherry Institute of Medical Sciences, Pondicherry, India, Tel: 04132651111; E-mail: kirencm@yahoo.com

Received February 24, 2019; Accepted March 01, 2019; Published March 04, 2019

Citation: Sharma R, Kiran CM, Ramdas A (2019) Morphological Alterations in Megakaryocytes in Bone Marrow Aspirate of Thrombocytopenia Cases. J Blood Lymph 9: 235

Copyright: $\odot 2019$ Sharma R, et al. This is an open-access article distributed unde the terms of the Creative Commons Attribution License, which permits unrestricted use, distribution, and reproduction in any medium, provided the original author and source are credited. 


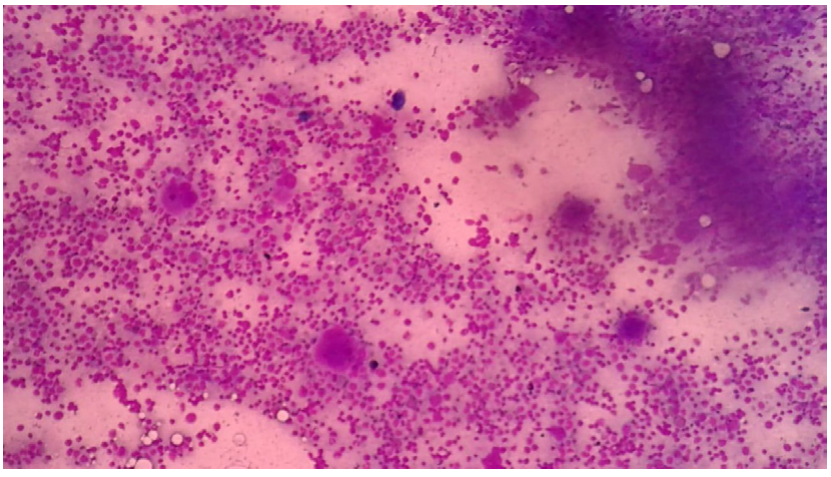

Figure 1: Increased number of megakaryocytes (Leishman, 4X).

scant bluish cytoplasm and lacking lobulation of the nucleus which occupied most of the cell [4]. Dysplastic megakaryocytes were defined as those with single/multiple separate nuclei. Micro-megakaryocytes were defined as megakaryocytes whose size was that of a large lymphocyte/ monocyte and which had a single/bilobed nucleus [2].

The megakaryocytes were considered to show platelet budding if there was budding of cytoplasmic processes from their surfaces. Hypogranular forms were defined as megakaryocytes with pale grey or water clear cytoplasm and sparse or no granules [2].

\section{Results}

Total of 100 cases were included in the study. Out of 100 cases of bone marrow aspiration, 78 cases were of thrombocytopenia, 6 cases were of thrombocytosis, and 16 cases were of normal platelet count. The most common cause for which bone marrow aspiration was sought was pancytopenia (30), followed by ITP (15), PUO (12), Anaemia (10), Bicytopenia (8), Hepatosplenomegaly (3), Lymphoproliferative disorder (5), GMCSF treatment (1), Myeloproliferative neoplasm; MPN (3) Tuberculosis MM (4 cases each), MDS (2), AL (2), MGUS (1).

Out of 30 cases of pancytopenia, 15 (50\%) showed increased no. of megakaryocytes, hypolobated forms seen in 9 cases (30\%), immature forms and emperipolesis seen in 7 cases $(23 \%)$, micromegakaryocytes seen in 6 cases $(20 \%)$.

All 15 cases of immune thrombocytopenia showed increase in number of megakaryocytes (Figure 2). Immature forms were seen in 14 cases $(93 \%), 10(66 \%)$ showed dysplastic forms, 10 (66\%) showed bare nuclei and $6(40 \%)$ showed emperipolesis. Out of 12 cases of fever, 5 (41\%) showed increase in number of megakaryocytes, 5 (41\%) showed immature forms, 3 (25\%) showed emperipolesis and 2 (16\%) showed dysplastic changes (Figure 3).

\section{Discussion}

Bone marrow aspiration is done for various clinical conditions, out of which thrombocytopenia is the most common. Megakaryocytes are unique cells which display single polypoid nucleus in which DNA keep on multiplying itself without cytoplasmic division (Figure 4). After achieving full maturations, megakaryocytes deform and their internal membrane spread out to form long extension and eventually platelets are shed out [5]. A defect in any stage of megakaryopoiesis can lead of dysmegakaryopoiesis and thrombocytopenia.

Dysplastic megakaryocytes are seen in MDS, but can also be seen in ITP, Infection Associated Thrombocytopenia (IAT), hypersplenism, aplastic anaemia, Acute myeloid leukemia, Acute Lymphoblastic

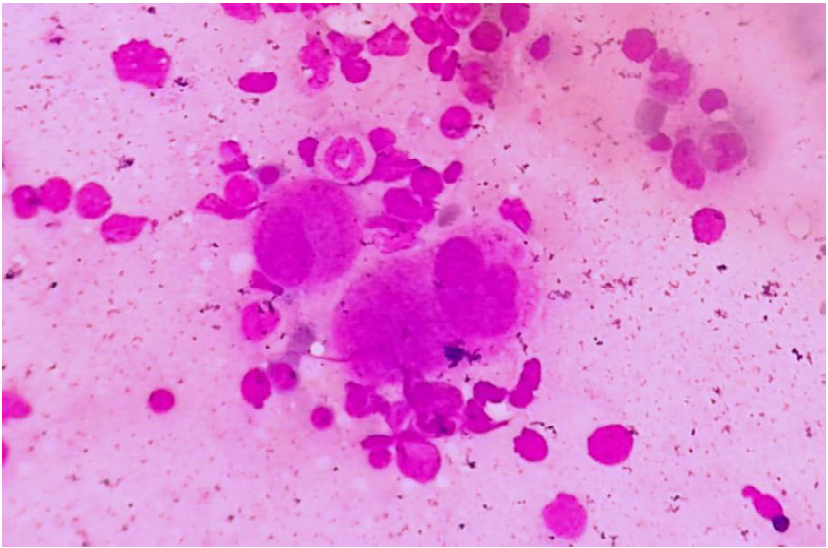

Figure 2: Hypolobated megakaryocytes (Leisman, 10X).

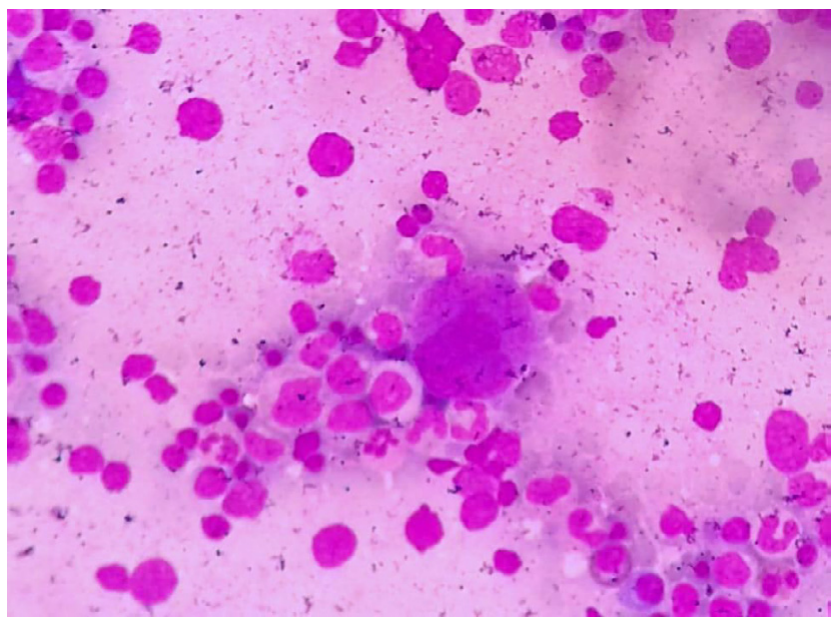

Figure 3: Immature megakaryocytes (Leishman, 10X).

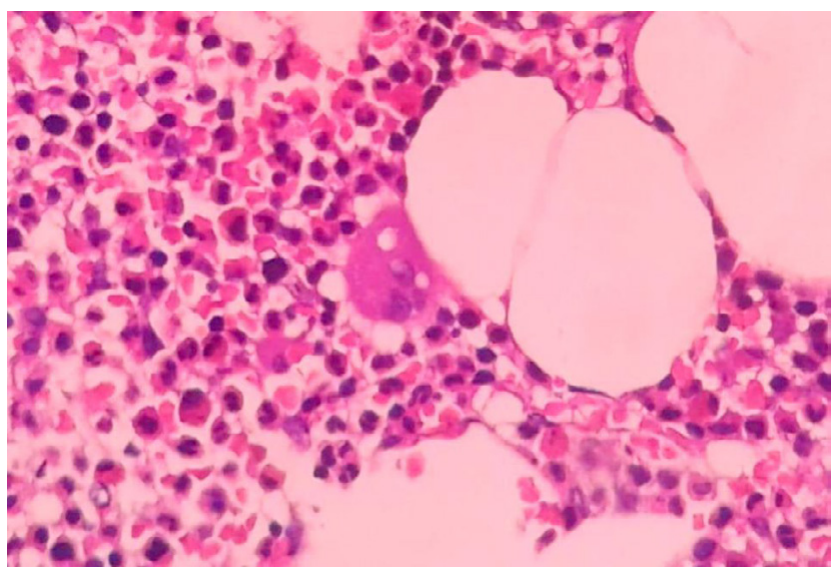

Figure 4: Cytoplasmic vacuoles (H\&E, 10X)

leukemia, bone marrow metastasis, blastic crisis of CML [2]. In all these diseases thrombocytopenia can be observed but mechanism can be different. The cause of thrombocytopenia in MDS and ITP is known to because of premature death. In MDS, megakaryocytes generally show features of necrosis like Programmed Cell Death (PCD) whereas 
Citation: Sharma R, Kiran CM, Ramdas A (2019) Morphological Alterations in Megakaryocytes in Bone Marrow Aspirate of Thrombocytopenia Cases. J Blood Lymph 9: 235.

Page 3 of 3

in ITP megakaryocytes show predominently apoptosis like PCD [5].

Emperipolesis with lymphocytes along with nucleated RBC and cytoplasmic vacuolations reflects increased megakaryocytes turnover, apoptosis and paraapoptosis [2]. In ITP 70\% of cases showed emperipolysis and $60 \%$ of cases showed cytoplasmic vacuolations. In case of ITP, the megakaryocytes are increased in number. Other morphological alterations like smooth forms with single nuclei, scanty cytoplasm are common Megaloblastic anaemia (6 cases), megakaryocytes were usually hyperlobated and had fine stippled chromatin than normal megakaryocytes. This is due to delayed DNA maturation than cytoplasmic maturation [6]. The most common dysplatic feature feature observed was multiple separate nuclei which was observed in almost all cases corresponding to Ashalatha et al. [7] study which showed $100 \%$ cases of megaloblastic anaemia showed dysplastic features. In contrast Muhury et al. [6] and Choudhary et al. [2] showed $75 \%$ and $52 \%$ cases with megakaryocytes with dysplastic features.

All 15 cases of ITP showed increase in number of megakaryocytes. In the study by Choudhary et al. [2] showed $91 \%$ cases of ITP with increased number of megakaryocytes whereas Muhury et al. found it in $94.7 \%$ of the case. Jubelirer et al. [8] also found increased megakaryocyte count in $95.3 \%$ of the cases of ITP. This could be due to stimulation of the marrow megakaryocytes to synthesize platelets at an increased rate due to immunemediated platelet destruction in the spleen and other reticulo-endothelial tissues [6].

In MDS, micromegakaryocytes are common and in this study both 2 cases had micromegakaryocytes in the bone marrow.

One case was of aplastic anaemia in which megakaryocytes were markedly decreased in number. In aplastic anaemia, reason for decreased platelet is because of bone marrow suppression and significant inhibition of nucleic acid synthesis in megakaryocytes. 1 case was of acute leukemia showed decreased number of megakaryocytes and showed dysplastic features. This could be due to defective differentiation of megakaryocyte to mature polyploidy cells [9].

In case of hypersplenism (3), all cases had increased megakaryocytes. This could be due to compensatory mechanism. Other parameters were not altered significantly. Emperipolysis process happens due to transmegakaryocyte route of some of the cells to outer in circulation and suggest megakaryocyte as a component of marrow blood barrier [6].

Cytoplasmic vacuolations is because of apoptosis and paraapoptosis. Another theory is auotophagy to maintain the cell metabolism and nutritional deficiency due to increased megakaryopoiesis [2,6].
5 cases of lymphoproliferative disorder, out of which 3 showed decreased megakaryocytes. Two cases were of MDS and both showed dysplastic features like hypogranular forms, multinucleation.

The limitation of this study was that we had only two cases of MDS and we could not find out the $\mathrm{p}$ value of dysplastic changes in megakaryocytes in MDS and non MDS conditions for comparison purpose. However in this study we have seen and descibe the various morphological changes in the megakaryocytes in bone marrow in thrombocytopenia cases.

\section{Conclusion}

Most common cause of thrombocytopenia in this study was pancytopenia followed by ITP. Dysplastic features were seen in MDS but also present in other conditions like megaloblastic anaemia acute leukemia. One should not go directly straight away to diagnosis of MDS but one should consider the other clinical parameters and percentage of dyspoietic forms before giving the probable diagnosis of MDS.

\section{References}

1. Patel SR, Hartwig JH, Italiano JE (2005) The biogenesis of platelets from megakaryocyte proplatelets. J Clin Invest 115: 3348-3354.

2. Muhury M, Mathai AM, Rai S, Naik R, Pai MR, et al. (2009) Megakaryocytic alterations in thrombocytopenia: A bone marrow aspiration study. Indian $J$ Pathol Microbiol 52: 490-494

3. Houwerijl EJ, Blom NR, van der Want JJ, Esselink MT, Koornstra JJ, et al. (2004) Ultrastructural study shows morphologic features of apoptosis and paraapoptosis in megakaryocytes from patients with idiopathic thrombocytopenic purpura. Blood 103: 500-506

4. McKenzie SB (1996) Textbook of hematology (2ndedn). Pennsylvania Willaims and Wilkins

5. Houwerzij EJ, Blom NR, van der Want JJ, Vellenga E, de Wolf JT (2006) Megakaryocytic dysfunction in myelodysplastic syndromes and idiopathic thrombocytopenic purpura is in part due to different forms of cell death Leukemia 20: 1937-1942.

6. Choudhary PK, Sing SK, Basnet RB (2013) Study of megakaryocytes in bone marrow aspiration smears in patients with thrombocytopenia. Journal of Pathology of Nepal 3: 476-481.

7. Ashalatha N, Netravathi P, Ragupathi A, Nagarajappa A (2010) Hemogram and bone marrow morphology in cases of pancytopenia. Internet J Lab Med 4

8. Jubelirer SJ, Harpold R (2002) The role of the bone marrow examination in the diagnosis of immune thrombocytopenic purpura: Case series and literature review. Clin Appl Thromb Hemost 8: 73-76.

9. Brandt L, Levan G, Mitelman F, Sjogren U (1974) Defective differentiation of megakaryocytes in acute myeloid leukemia. Acta Med Scand 196: 227-230. 